Original Research Paper

\title{
Abnormalities in Ultrasonic (C-Scan) Images of Composite Structures: Impact Damaged Versus Hole Damaged
}

\author{
Mahmoud Zaki Iskandarani \\ Faculty of Engineering, Al-Ahliyya Amman University, Amman, Jordan
}

Article history

Received: 09-05-2019

Revised: 13-06-2019

Accepted: 22-07-2019

Email: m.iskandarani@ammanu.edu.jo

\begin{abstract}
This paper is about applying image processing to detect different types and levels of damage in composite structures using in C-scan images produced by ultrasonic testing. This method plays an important role as it can detect any discontinuities or flaws in the specimen. To analyze such critical image in an, automated inspection environment an image processing method that correlates Threshold values to Gradient Field values is used to extract important information from the c-scan images in order to detect abnormalities that may exist. The paper presents an improved approach to composite damage characterization using Threshold Level Variation as an input variable to Gradient Fields. Furthermore, the detailed results showed an important dependency between what can be detected and the used threshold level. At each level certain abnormalities appeared. Setting threshold value is proved to be a function of image type and quality, purpose and application.
\end{abstract}

Keywords: Gradient Norm, Edge Detection, Gray Level Mapping, Segmentation, Threshold, C-Scan, Similarity Detection, Damage Detection, Composites

\section{Introduction}

Composite laminated has a marked impact on engineering as many industries are attempting to produce lightweight material with best quality. To achieve highest quality in production automated damage detection is the optimum way to solve the problem.

Inspection process is the most crucial part in a production line of many industries in order to reduce defective products produced during the manufacturing process. Thus, Non-Destructive Testing (NDT) is introduced to overcome the DT method that had been known as a time consuming inspection process.

One of the main tasks in composite structures maintenance and extension of service life of components is to nondestructively test the produced component critical elements. Many elements of functioning composite structure components in applications such as vehicles and aircrafts are vulnerable to even smallest impact that produces a hardly visible damage. One of the primary testing techniques used for composite structure inspection is an ultrasonic C-Scan technique which application results in planar images of emitted/received wave attenuation and a time of flight map (Kroworz and Katunin, 2018; González et al., 2017).
Ultrasonic C-Scan is easy to use and provides high accuracy image measurement with proved reliability. The acquired image through the C-Scan technique is full of features that can be extracted, which provide critical information.

Segmentation of C-Scan images using region growing technique is an important approach because of its ability to analyze structures. Region based segmentation of C-Scan images can be used in many industries and applications, such as automotive and aerospace. Damage detection in composite structures through visualization, defect detection, fiber breakage detection and composite structure penetration are few examples of cases that can be captured and analyzed using region growing and segmentation. Regions based intensity is the most challenging task.

Segmentation is critical step in any image analysis process where the image output is some detailed description of the object status. The segmentation process uses measured image features with correlative and associative regions that holds such features to enable correct detection and classification of damage.

Image segmentation techniques employs different approaches such as thresholding, boundary-based, region-based and contouring methods. 
Boundary-based techniques are based on the pixel characteristic which changes at the boundary between two regions using edge detection technique are used to produce curves separating boundaries between adjacent regions. However, this approach suffers difficulty in edge conversion to close boundary. Region based segmentation techniques are complementary to boundary-based approach.

Contour techniques are based on energy minimization principles, effective for finding regions with high frequency variation.

Thresholding based techniques relies on the brightness parameter called threshold in order to perform image segmentation. Thresholding method works best in images which has a bimodal distribution.

Thresholding is a simple but effective technique that isolates areas or regions of interest; its applications covering medical, automotive and aerospace, among other areas of applications. Thresholding selection techniques can be classified into two categories: bi-level and multi-level. A variety of thresholding approaches exist for image segmentation, ranging from conventional methods to intelligent techniques (Ma and Liu, 2016; Iskandarani, 2017; Suganya et al., 2013; Jung et al., 2017; Geng et al., 2015; Santhi and Wahida Banu, 2015).

\section{Background}

Ultrasound waves have frequencies above the upper limit of audibility which is approximately $20 \mathrm{KHz}$, although the range of 1 to Ultrasound travels in the mediums in the form of stress waves. Several modes of propagation are possible in material structures such as:

- Compressional waves

- Shear waves

- Surface waves

- $\quad$ Plate and Rod waves

The interaction between the ultrasound wave and defects within the tested structure is a function of the defect size, defect orientation and propagation mode among other factors

A material boundary constitutes a change in the acoustic impedance. At the interface between two different environments, some sound is reflected and the rest is transmitted across the interface. The relative amplitude and phases of these waves depend upon the change in properties across the boundary. The nature of wave-material interaction is complicated as one kind of incident wave can result in the generation of other types within a structure.

These types of waves tend to get modified by the encountered boundaries, by the material itself and the presence of defects, which in non-uniform composite structures and/or damaged composites will most of the time result in the creation of edges within the fibermatrix system. Consequently, segmentation will occur and subdivides the composite image into regions. The number of regions formed as a result of segmentation depends on the type of damage a structure suffered. The segmented image is a function of both discontinuity and uniformity. Both factors can be used to establish similarity and level of damage as a result of abrupt change or edges (Murat and Rahman, 2017; Putkis et al., 2016; Murat et al., 2016; Murat and Fromme, 2016).

Due to the complex nature of barely visible impact damage occurrence it is difficult to analyze the resulting $\mathrm{C}$-Scan images. Therefore, it is necessary to implements image processing techniques and modify existing algorithms to accommodate critical analysis of resulting images.

Image processing algorithms are directed towards the detection of features through labeling, for later analysis and for quality control through Non-Destructive Testing (NDT). Hence, image segmentation aims to group pixels within meaningful regions. Commonly, gray levels belonging to an affected region, are substantially different from those featuring the unaffected regions.

This work proposes an image processing algorithm for evaluation of such damage distribution in composite structures. The presented research investigates the effect of threshold variation on damage detection as applied to C-Scan images of composite structures. The algorithm is based on multilevel thresholding of RGB images resulting from $\mathrm{C}$-Scan testing with correlative displacement of gradient fields associated with damaged areas of composite structures. Using the proposed algorithm an extraction of damage visualization from a C-Scan image is carried out (Atta and Abdel-Kader, 2015; Xu et al., 2015; Singh et al., 2015; Guo et al., 2018; Lee et al., 2017; Furnari et al., 2017; Huang et al., 2015; Iskandarani, 2018).

The main contribution of this work is to enable distinction between impact damaged composite structures and penetrated composite structures, where penetration of objects can compromise the integrity of the device and can have catastrophic outcomes as in the automotive and aviation areas.

The developed approach will allow supporting diagnosing of damaged composite structures due to impact and due to structure penetration as in the case of drilling. The paper is structured to cover used techniques, obtained images and application of Gradient Field with variable Threshold technique to accurately establish damage type and level. 


\section{Materials and Methods}

The main objective is to detect damage and uniformity variation through gradient application to captured images. The ultrasonic testing system uses water as an interface where the specimen is immersed in a water tank and the transducer is held in position by a manipulator so that the orientation and distance from the specimen can be adjusted. The transducer is scanned relative to the specimen automatically.

In this type of inspection, a complex scanning system is used such that the transducer is scanned in a plane parallel to the sample in a rectilinear raster pattern. The output is interfaced to a computing system allowing full color graphics output to be post processed. The used equipment comprised:

(1). Ultrasonic Immersion Tank system, which included

(a) Immersion Tank of approximate dimensions of $1 \times 0.5 \times 0.4 \mathrm{~m}$ with 3 motorized $3-\mathrm{D}$ axis

(2). Power controller cabinet with 3 power modules, and $7.5 \mathrm{MHz}$ immersion probes on two axes manipulator

(b) Computing system box with pulser/receiver card and processing software

The C-Scan system was set up to measure peak amplitude of the back wall echo through line scanning of the immersed $5 \mathrm{~mm}$ thick woven glass composite components in the water tank. Two types of samples tested:

- Impact damaged samples

- Drilled samples

The obtained RGB images (RGB images are M-byN-by-3) converted to Black and White (BW) by converting each image to Grayscale format first and then converting each Grayscale image to binary image by thresholding. The output binary image BW has values of 1 (white) for all pixels in the input image with luminance greater than Specified LEVEL, which is between 0 and 1 and 0 (black) for all other pixels.

The Gradient and Scaled Gradient calculated using the following expressions:

$$
\begin{gathered}
\operatorname{Grad}_{\text {Mag }}=\left[\sqrt{\left(d x_{\text {image }}\right)^{2}+\left(d y_{\text {image }}\right)^{2}}\right] \\
\operatorname{Grad}_{\text {Magscaled }}=\left[\frac{\operatorname{Grad}_{\text {Mag }}}{M A X\left(\operatorname{Grad}_{\text {Mag }}\right)}\right]
\end{gathered}
$$

A two components of the same Woven Glass material manufactured under exactly same conditions, one subjected to impact damage at 29Joules and the other to drilling machine to produce a hole through the component. It is assumed the structural and surface variation among them is minimal allowing for comparison.

\section{Results}

\section{Impact Damage Case}

Figures 1 to 5 show detected impact damage in CScan images of Woven Glass composite structure by threshold variation as a condition for conversion from RGB BW through Gray levels. The impact energy is 29 Joules delivered via a dropped vertical load. Figure 6 to 8 show other surface abnormalities when the threshold level exceeds a critical and optimized value that is used to isolate the impact damaged area from the rest of the component. Figure 9 indicates the end of ability to detect the discovered impact damage due to threshold new value.

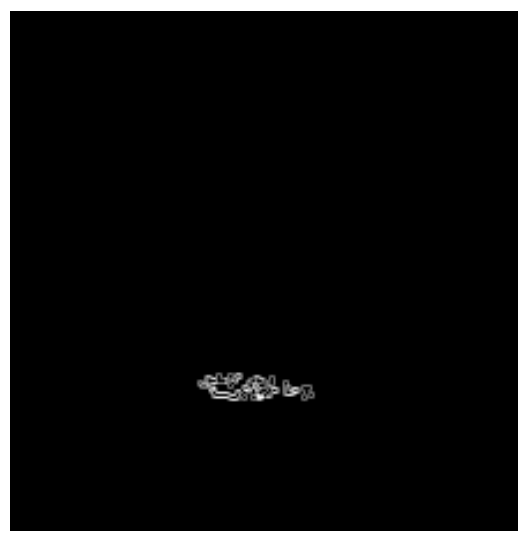

Fig. 1: Gradient of C-Scan Image: Impact Damage $(0.15$ Threshold)

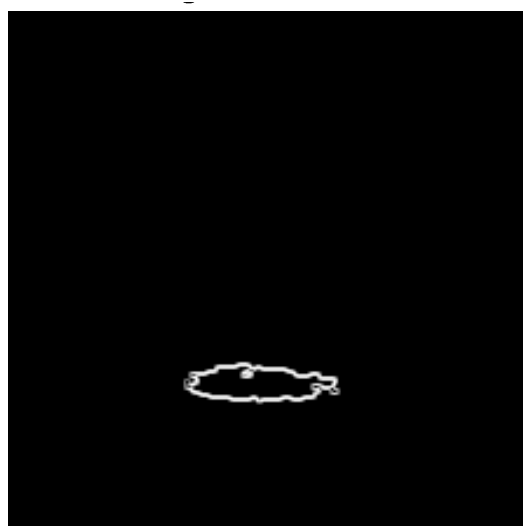

Fig. 2: Gradient of C-Scan Image: Impact Damage (0.2 Threshold) 


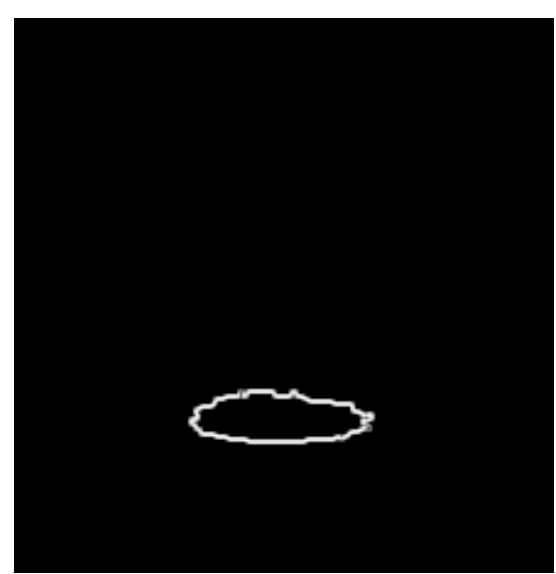

Fig. 3: Gradient of C-Scan Image: Impact Damage $(0.25$ Threshold)

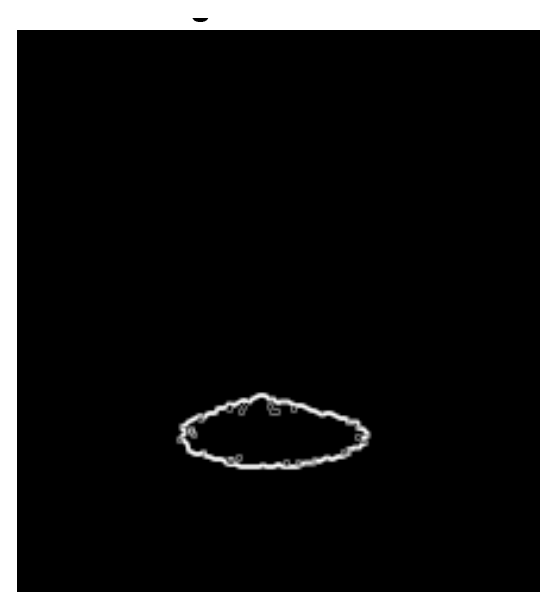

Fig. 4: Gradient of C-Scan Image: Impact Damage $(0.3$ Threshold)

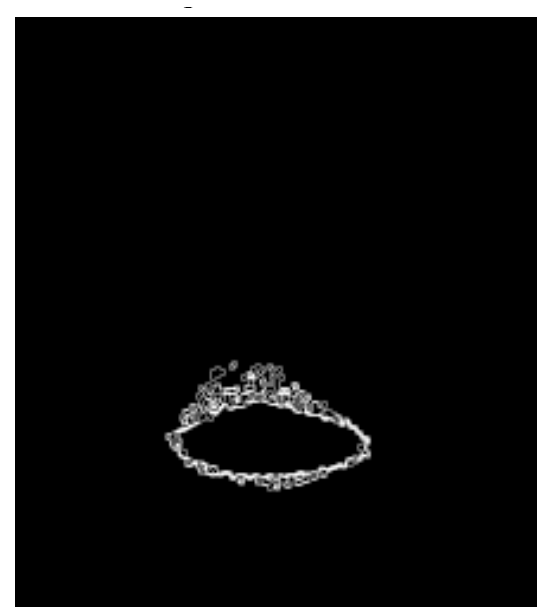

Fig. 5: Gradient of C-Scan Image: Impact Damage $(0.35$ Threshold)

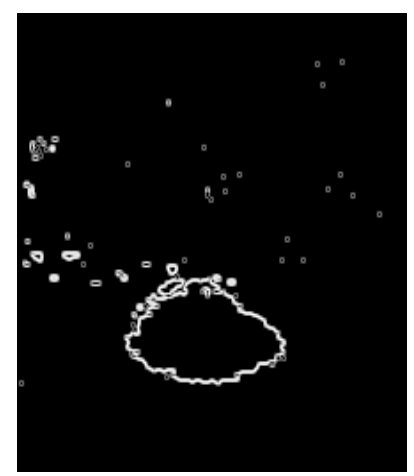

Fig. 6: Gradient of C-Scan Image: Impact Damage and Surface Abnormalities (0.4 Threshold)

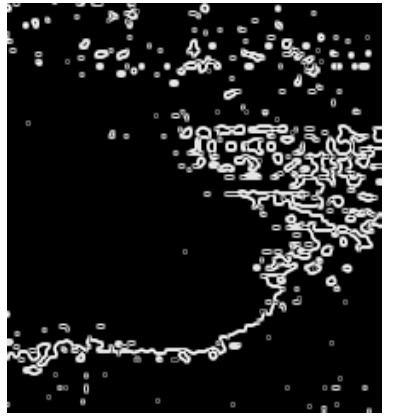

Fig. 7: Gradient of C-Scan Image: Surface Abnormalities (0.45 Threshold)

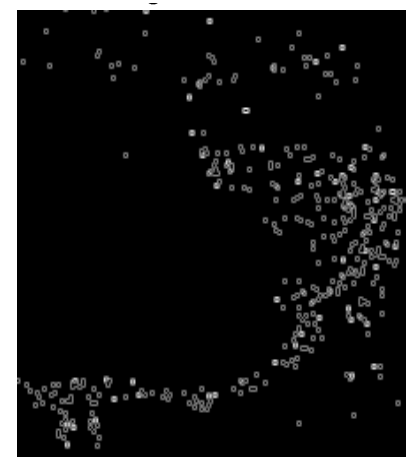

Fig. 8: Gradient of C-Scan Image: Surface Abnormalities (0.5 Threshold)

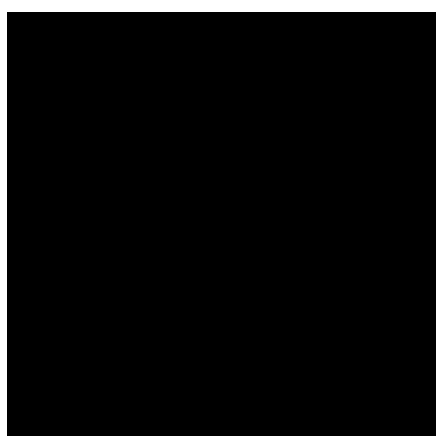

Fig. 9: Gradient of C-Scan Image: End of impact Damage Detection (0.55 Threshold) 


\section{Hole Through Case}

Figures 10 and 11 show detected impact damage in C-Scan images of Woven Glass composite structure by threshold variation as a condition for conversion from RGB BW through Gray levels. Figure 12 to 14 show the presence of surface abnormalities in addition to the hole, which starts to diminish in size. This occurs when the threshold level exceeds a critical and optimized value that is used to isolate the impact damaged area from the rest of the component. Figure 15 and 16 show a fixed but reduced through hole detected but without surface abnormalities as a function of threshold value. The new hole details and size stays constant up to threshold value 0.80 , after that it starts to disappear until it reaches a very low value as shown in Fig. 16, after which no hole detection is possible at threshold 1.0 .

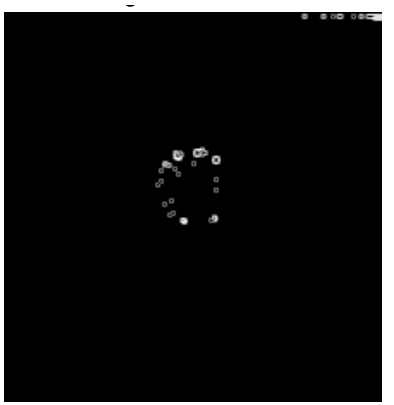

Fig. 10: Gradient of C-Scan Image: Hole Through (0.30 Threshold)

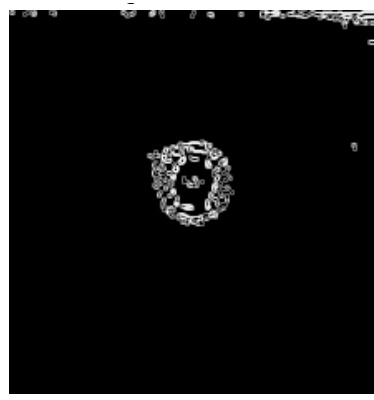

Fig. 11: Gradient of C-Scan Image: Hole Through $(0.35$ Threshold)

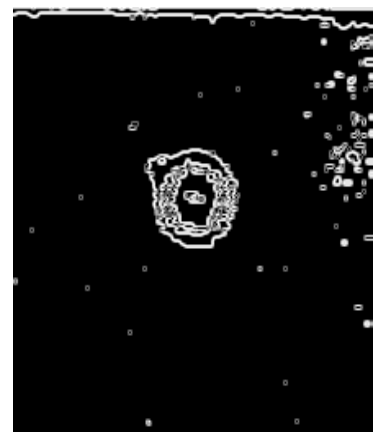

Fig. 12: Gradient of C-Scan Image: Hole Through and Appearance of Surface Abnormalities ( 0.40 Threshold)

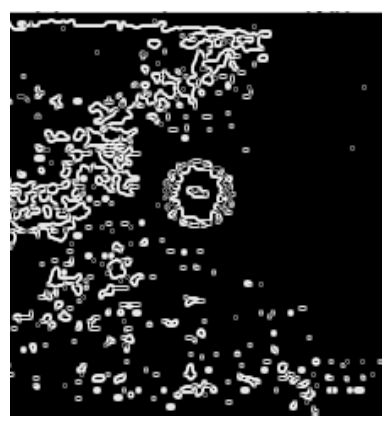

Fig. 13: Gradient of C-Scan Image: Reduced Hole Size (Loss of Details) and the Appearance of Surface Abnormalities ( 0.45 Threshold)

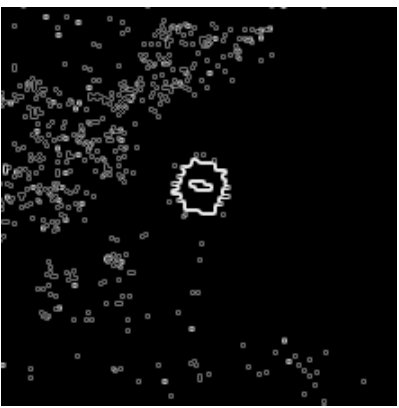

Fig. 14: Gradient of C-scan Image: Reduced hole size (Loss of Details) and the appearance of surface abnormalities ( 0.50 Threshold)

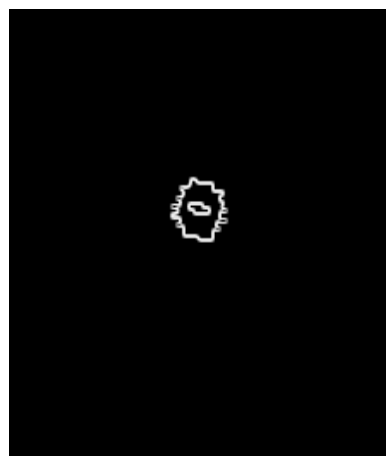

Fig. 15: Gradient of C-Scan Image: Fixed new Hole Size (Loss of Details at 0.55 Threshold))

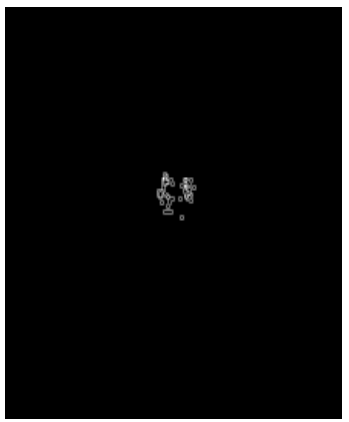

Fig. 16: Gradient of C-Scan Image: Reduced Hole Size (Loss of Details at 0.95 Threshold) 


\section{Analysis and Discussion}

\section{Impact Damage Case:}

Table 1 show the effect of varying the threshold level on the pixel distribution of the C-Scan image of impact damaged Woven Glass composite structure. The structure suffered a 29 Joules of impact energy delivered through a vertically launched load.

\section{Hole through Case:}

Table 2 show the effect of varying the threshold level on the pixel distribution of the C-Scan image of a drilled composite structure,

From Tables 1 and 2, we observe the following:

1. The initial Threshold level at which an impact damage is detected is 0.15 , while for Drilled Hole is 0.30 .

2. The final Threshold value after which no impact damage can be detected is 0.35 , and for Drilled Hole is 0.95

3. The Threshold interval for impact damage is $\{0.15$, $0.35\}$, while for Drilled hole, there are two intervals separated by external surface irregularities: $\{0.30$, $0.35\}$ and $\{0.55,0.95\}$

4. For both components, surface abnormalities appear in the interval $\{0.40,0.5\}$, which is consistent with the fact that they undergone same manufacturing process, and the difference is due to each one being subject to different type of external damage and handling

From the observations, characteristic matrix can be highlighted for both cases shown in Fig. 17 to 22:
Impact Damaged Case:

$\left[\begin{array}{cccc}0.15 & 48170 & 198 & 32 \\ 0.20 & 48086 & 248 & 66 \\ 0.25 & 48062 & 274 & 64 \\ 0.30 & 47994 & 302 & 104 \\ 0.35 & 47542 & 662 & 196\end{array}\right]$

Hole through Case:

$$
\begin{aligned}
& {\left[\begin{array}{cccc}
0.30 & 48170 & 200 & 30 \\
0.35 & 47102 & 1025 & 273
\end{array}\right]} \\
& {\left[\begin{array}{cccc}
0.80 & 48098 & 248 & 54 \\
0.85 & 48102 & 244 & 54 \\
0.90 & 48142 & 184 & 74 \\
0.95 & 48256 & 124 & 20
\end{array}\right]}
\end{aligned}
$$

For the same component, which clearly will have same surface abnormalities, the detection of surface impact damage occurs at different threshold value than the one required to uncover the presence of hole in the component.

Also, the detected impact damage cycle will be affected. Hence we can introduce a discrimination function, which is used to distinguish a surface damage from damage caused by penetrating elements as shown in Equations (1) and (2):

$$
\begin{aligned}
& \text { Damage }=\sum_{k=0}^{255} \operatorname{Im} \text { age }\left(\text { Threshold }_{D}\right) \\
& +\sum_{k=0}^{255} \text { Surface }_{\text {Abnormalities }}\left(\text { Threshold }_{D}\right)
\end{aligned}
$$

\begin{tabular}{|c|c|c|c|c|}
\hline \multicolumn{5}{|c|}{ Impact damage pixel distribution } \\
\hline Threshold & Gray Levels & & & \\
\hline$\{0,1\}$ & 0 & 128 & 180 & 255 \\
\hline 0 & 48400 & 0 & 0 & 0 \\
\hline 0.05 & 48400 & 0 & 0 & 0 \\
\hline 0.1 & 48400 & 0 & 0 & 0 \\
\hline 0.15 & 48170 & 198 & 32 & 0 \\
\hline 0.2 & 48086 & 248 & 66 & 0 \\
\hline 0.25 & 48062 & 274 & 64 & 0 \\
\hline 0.3 & 47994 & 302 & 104 & 0 \\
\hline 0.35 & 47542 & 662 & 196 & 0 \\
\hline 0.4 & 47304 & 852 & 242 & 2 \\
\hline 0.45 & 42854 & 4256 & 1268 & 22 \\
\hline 0.5 & 46720 & 1615 & 58 & 7 \\
\hline 0.55 & 48400 & 0 & 0 & 0 \\
\hline 0.6 & 48400 & 0 & 0 & 0 \\
\hline 0.65 & 48400 & 0 & 0 & 0 \\
\hline 0.7 & 48400 & 0 & 0 & 0 \\
\hline 0.75 & 48400 & 0 & 0 & 0 \\
\hline 0.8 & 48400 & 0 & 0 & 0 \\
\hline 0.85 & 48400 & 0 & 0 & 0 \\
\hline 0.9 & 48400 & 0 & 0 & 0 \\
\hline 0.95 & 48400 & 0 & 0 & 0 \\
\hline 1 & 48400 & 0 & 0 & 0 \\
\hline
\end{tabular}

Table 1: Impact damage results 
Table 2: Hole through results

Hole through pixel distribution

\begin{tabular}{|c|c|c|c|c|}
\hline \multirow{2}{*}{$\begin{array}{l}\text { Threshold } \\
\{0,1\}\end{array}$} & \multicolumn{4}{|c|}{ Gray Levels } \\
\hline & 0 & 128 & 180 & 255 \\
\hline 0 & 48400 & 0 & 0 & 0 \\
\hline 0.05 & 48400 & 0 & 0 & 0 \\
\hline 0.1 & 48400 & 0 & 0 & 0 \\
\hline 0.15 & 48400 & 0 & 0 & 0 \\
\hline 0.2 & 48400 & 0 & 0 & 0 \\
\hline 0.25 & 48400 & 0 & 0 & 0 \\
\hline 0.3 & 48170 & 200 & 30 & 0 \\
\hline 0.35 & 47102 & 1025 & 273 & 0 \\
\hline 0.4 & 45540 & 2134 & 562 & 164 \\
\hline 0.45 & 41136 & 5361 & 1673 & 230 \\
\hline 0.5 & 46044 & 2191 & 147 & 18 \\
\hline 0.55 & 48098 & 248 & 54 & 0 \\
\hline 0.6 & 48098 & 248 & 54 & 0 \\
\hline 0.65 & 48098 & 248 & 54 & 0 \\
\hline 0.7 & 48098 & 248 & 54 & 0 \\
\hline 0.75 & 48098 & 248 & 54 & 0 \\
\hline 0.8 & 48098 & 248 & 54 & 0 \\
\hline 0.85 & 48102 & 244 & 54 & 0 \\
\hline 0.9 & 48142 & 184 & 74 & 0 \\
\hline 0.95 & 48256 & 124 & 20 & 0 \\
\hline 1 & 48400 & 0 & 0 & 0 \\
\hline
\end{tabular}

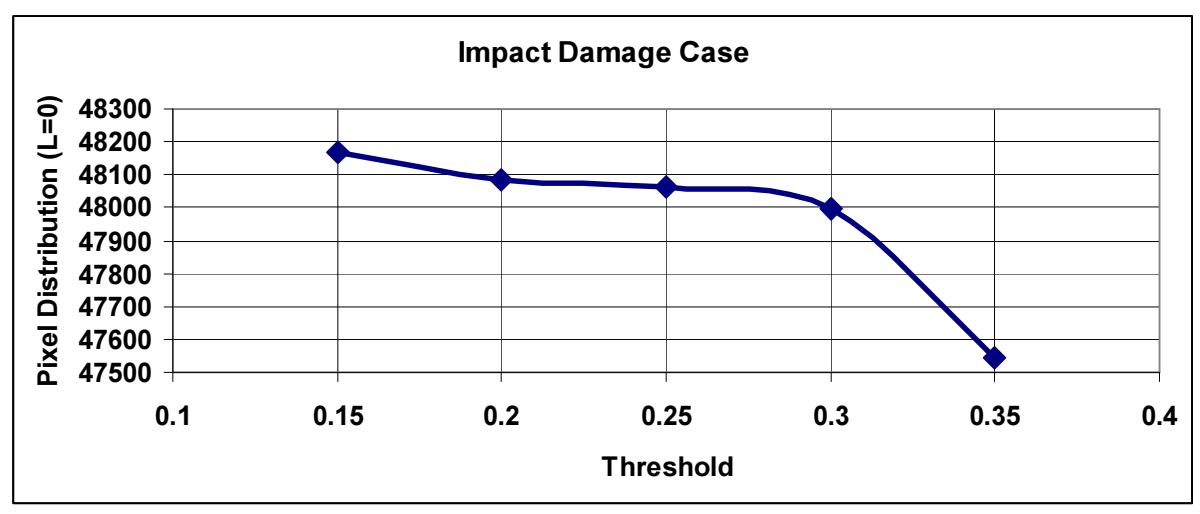

Fig. 17: Pixel distribution as a function of Threshold

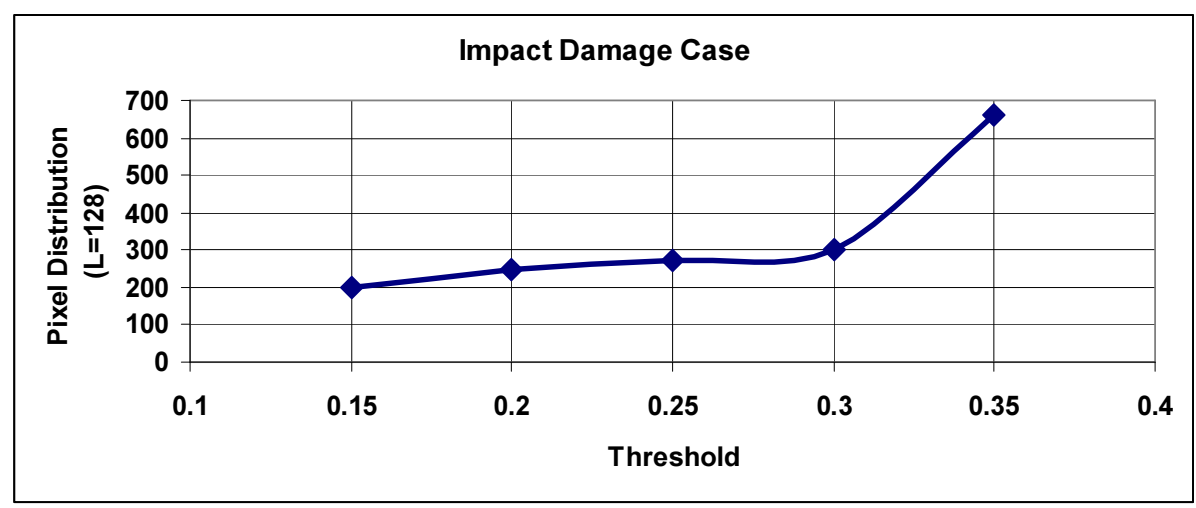

Fig. 18: Pixel distribution as a function of Threshold 


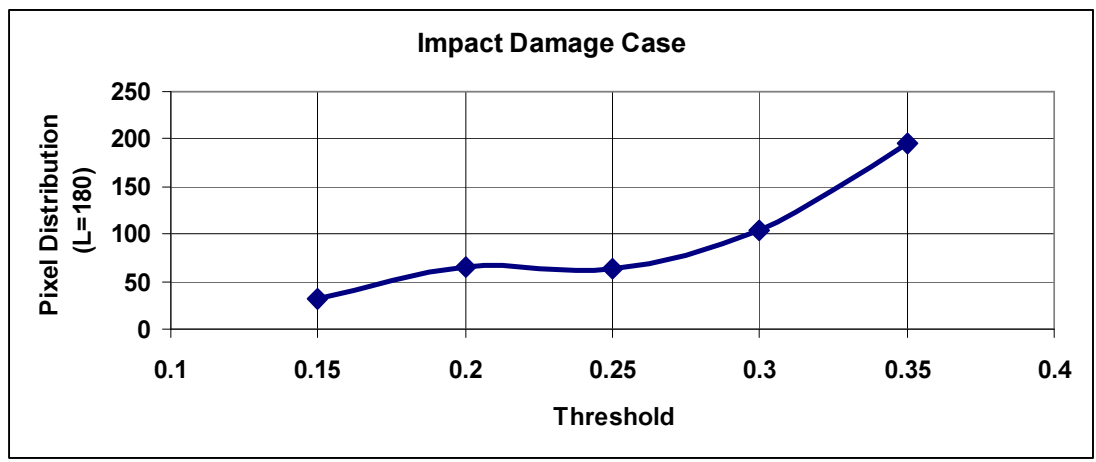

Fig. 19: Pixel distribution as a function of Threshold

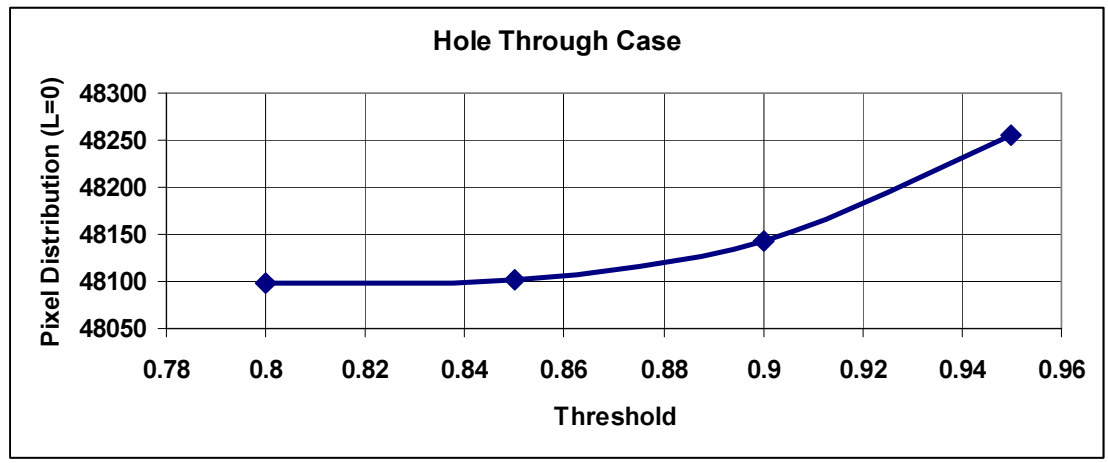

Fig. 20: Pixel distribution as a function of Threshold

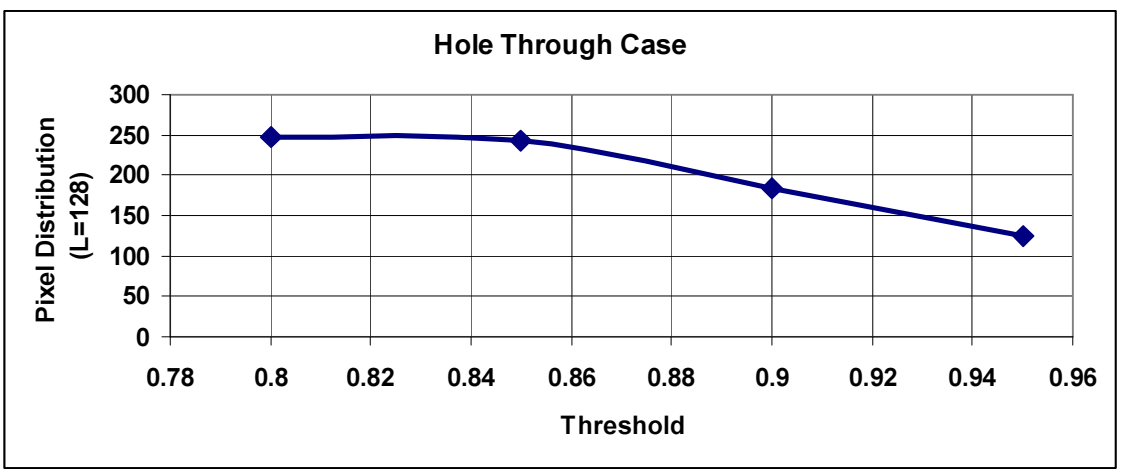

Fig. 21: Pixel distribution as a function of Threshold

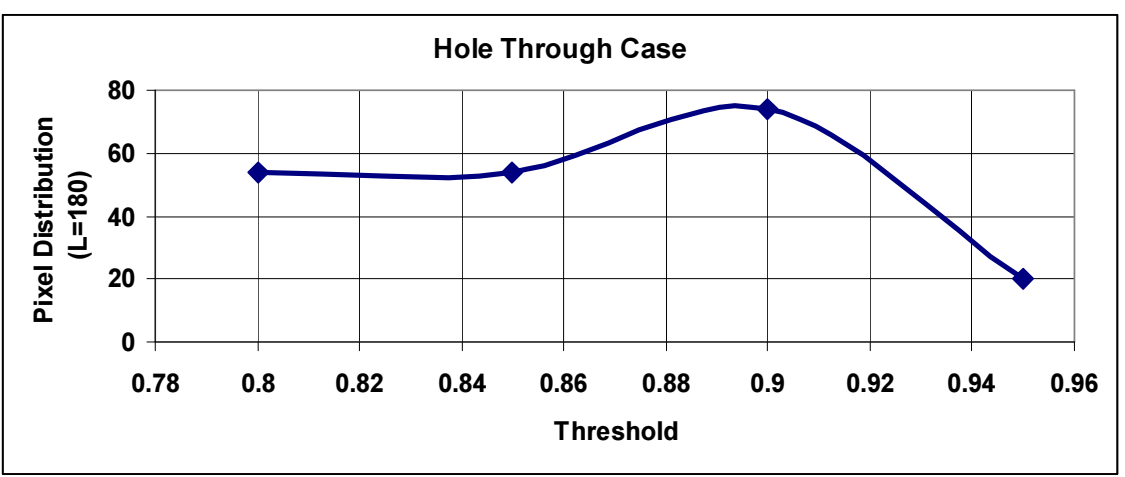

Fig. 22: Pixel distribution as a function of Threshold 
Hole $=\sum_{k=0}^{255} \operatorname{Im}$ age $\left(\right.$ Threshold $\left._{H}\right)$

$+\sum_{k=0}^{255}$ Surface Abnormalities $_{k}\left(\right.$ Threshold $\left._{H}\right)$

The threshold values can be expressed as in Equations (3) and (4):

Threshold $_{H}=Q .\left(\right.$ Threshold $\left._{D}\right)$

Threshold $_{D}=\left(\frac{\text { Threshold }_{H}}{Q}\right)$

where, $Q$ is a material related factor and could be obtained through different materials testing. Substituting Equation (3) into Equation (2) and Equation (4) into Equation (1):

$$
\begin{aligned}
& \text { Hole }=\sum_{k=0}^{255} \operatorname{Im} \operatorname{age}\left(Q .\left(\text { Threshold }_{D}\right)\right) \\
& +\sum_{k=0}^{255} \text { Surface Abnormalities }_{k}\left(Q .\left(\text { Threshold }_{D}\right)\right)
\end{aligned}
$$

Damage $=\sum_{k=0}^{255} \operatorname{Im}$ age $\left(\frac{\text { Threshold }_{H}}{Q}\right)$

$+\sum_{k=0}^{255}$ Surface Abnormalities $_{k}\left(\frac{\text { Threshold }_{H}}{Q}\right)$

At optimum Threshold, the surface abnormalities will not show, reducing Equations (5) and (6) to:

Hole $=\sum_{k=0}^{255} \operatorname{Im} \operatorname{age}\left(Q \cdot\left(\right.\right.$ Threshold $\left.\left._{D}\right)\right)$

Damage $=\sum_{k=0}^{255} \operatorname{Im}$ age $\left(\frac{\text { Threshold }_{H}}{Q}\right)$

Variation of threshold will affect pixel distribution, consequently resulting in zooming on the damaged or affected areas in the tested component and segmenting bad parts from good parts of the structure through clustering of similarity values, which results in regions separated by edges. Pixel distribution in an image as a function of threshold can be expressed as:

$\operatorname{Im}$ age $=f($ Threshold $)=\sum_{i=0}^{255} P_{i}$

In the obtained C-Scan images, the change in four main levels as a function of threshold control damage and abnormalities detection cycle, hence:

$\operatorname{Im}$ age $=f($ Threshold $)=\sum P_{0}+P_{128}+P_{180}+P_{255}$
Also, Surface Abnormalities appear in an image as a function of threshold. They appear populating certain levels. In the case of the C-Scan images in this work, Surface Abnormalities appear when Level 255 is populated. Taking this into account and using Equations (9), (1), and (2) results in:

$$
\begin{aligned}
& \text { Damage }=\left[\sum_{\text {Threshold }=0}^{1.0}\left(P_{0}+P_{128}+P_{180}\right)\right]+\left[P_{255}\right] \\
& \text { Hole }=\left[\sum_{\text {Threshold }=0}^{1.0}\left(P_{0}+P_{128}+P_{180}\right)\right]+\left[P_{255}\right]
\end{aligned}
$$

From Equations (10) and (11), three main cases are proved:

\section{Case 1: No Defect (Damage or Hole) Detected:}

In this case, $\mathrm{P}_{0}$ is the only populated level, so no damage or hole can be detected.

\section{Case 2: Surface Abnormalities Detected:}

In this case and as both samples are from the same manufactured patch, with similar surface properties, only affected by the type of exposure to impact and drilling instrument, Surface Abnormalities only appear as stated in Equations (12) and (13) over a range of specific threshold values and disappears otherwise:

$$
\begin{aligned}
& \text { Damage }=\left[\sum_{\text {Threshold }=0.4}^{0.5}\left(P_{0}+P_{128}+P_{180}\right)\right]+\left[P_{255}\right] \\
& \text { Hole }=\left[\sum_{\text {Threshold }=0.4}^{0.5}\left(P_{0}+P_{128}+P_{180}\right)\right]+\left[P_{255}\right]
\end{aligned}
$$

\section{Case 3: Defect (Damage or Hole) is Detected:}

In this case, all levels but $\mathrm{P}_{255}$ are populated. Equation (10) is reduced to:

Damage $=\left[\sum_{\text {Threshold }=0.15}^{0.35}\left(P_{0}+P_{128}+P_{180}\right)\right]$

While Equation (11) is reduced to:

$$
\begin{aligned}
& \text { Hole }=\left[\sum_{\text {Threshold }=0.30}^{0.35}\left(P_{0}+P_{128}+P_{180}\right)\right] \\
& \text { Hole }=\left[\sum_{\text {Threshold }=0.55}^{0.95}\left(P_{0}+P_{128}+P_{180}\right)\right]
\end{aligned}
$$




\section{Conclusion}

This work showed a new approach with mathematical modelling to enable not only detection but also distinction between surface impact damage, surface abnormalities that are related to finishing, and deep cuts like holes in composites. The approach clearly shows how to detect and evaluate damage within woven glass composite components using gradient based image processing applied to $\mathrm{C}$-scan images.

The presented work could be further enhanced by deploying variable scanning frequencies and other imaging devices such as thermal imaging with intelligent correlation algorithms that enable multi-dimensional detection and characterization of damage.

\section{Ethics}

This article is original and contains unpublished material. The corresponding author confirms that all of the other authors have read and approved the manuscript and no ethical issues involved.

\section{References}

Atta, R. and R. Abdel-Kader, 2015. Brightness preserving based on singular value decomposition for image contrast enhancement. Optik. Int. J. Light Electron Optics, Elsevier, 126: 799-803. DOI: $10.1016 /$ j.ijleo.2015.02.025

Furnari, A., G. Farinella, A. Bruna and S. Battiato, 2017. Distortion adaptive Sobel filters for the gradient estimation of wide angle images. J. Vis. Commun. Image R., 46: 165-175.

DOI: $10.1016 /$ j.jvcir.2017.03.019

Geng, P., X. Su, T. Xu and J. Liu, 2015. Multi-modal medical image fusion based on the multiwavelet and non-sub sampled direction filter bank. Int. J. Signal Proc. Image Proc. Pattern Recognition, 8: 75-84. DOI: 10.14257/IJSIP.2015.8.11.08

González, C., J. Vilatela, J. Molina-Aldareguía, C. Lopes and J. LLorca, 2017. Structural composites for multifunctional applications: Current challenges and future trends. Prog. Mater. Sci., 89: 194-251.

DOI: 10.1016/j.pmatsci.2017.04.005

Guo, Y., R. Wei and Y. Liu, 2018. Weighted gradient feature extraction based on multiscale sub-blocks for $3 \mathrm{~d}$ facial recognition in bimodal images. Informatics, 9: 529-551. DOI: 10.3390/info9030048

Huang, L., W. Zhao and Z. Jun Wang, 2015. An advanced gradient histogram and its application for contrast and gradient enhancement. Optik Int. J. Light Electron Optics, Elsevier, 31: 86-100.

DOI: 10.1016/j.jvcir.2015.06.007
Iskandarani, M., 2017. Correlating and modeling of extracted features from PVT images of composites using optical flow technique and weight elimination algorithm optimization [OFT-WEA]. J. Comp. Sci., 13: 371-379. DOI: $10.3844 /$ jcssp.2017.371.379

Iskandarani, M., 2018. Modelling of thermal storage in damaged composite structures using time displaced gradient field technique. Int. J. Advan. Comp. Sci. Applic., 9: 55-59. DOI: 10.14569/IJACSA.2018.090608

Jung, C., Q. Yang, T. Sun and Q. Hyoseob Song, 2017. Low light image enhancement with dual-tree complex wavelet transform. J. Visual Commun. Image Representation, 42: 28-36. DOI: $10.1016 /$ j.jvcir.2016.11.001

Kroworz, A. and A. Katunin, 2018. Non-destructive testing of structures using optical and other methods: A review. SDHM, 12: 1-17. DOI: $10.3970 /$ sdhm.2018.012.001

Lee, H., J. Jeon, J. Kim and S. Lee, 2017. Structure $\square$ texture decomposition of images with interval gradient. Computer Graphics Forum, 36: 262-274. DOI: $10.1111 /$ cgf.12875

Ma, L. and D. Liu, 2016. Delamination and fiberbridging damage analysis of angle-ply laminates subjected to transverse loading. Int. J. Imaging Systems Technol., 50: 3063-3075. DOI: $10.1177 / 0021998315615647$

Murat, B. and A. Rahman, 2017. Study of impact damage behavior in woven carbon fiber plates. Procedia Eng., 170: 47-54. DOI: 10.1016/J.PROENG.2017.03.009

Murat, B. and P. Fromme, 2016 Propagation and scattering of guided waves in composite plates with defects. Int. J. Automot. Mech. Eng., 13: 3728-3741. DOI: 10.15282/ijame.13.3.2016.15.0305

Murat, B., P. Khalili and P. Fromme, 2016. Scattering of guided waves at delaminations in composite plates. J. Acoust. Soc. Am., 139: 3044-3052. DOI: $10.1121 / 1.4953016$

Putkis, O., R. Dalton and A. Croxford, 2016. The anisotropic propagation of ultrasonic guided waves in composite materials and implications for practical applications. Ultrasonics, 65: 390-399.

DOI: 10.1016/j.ultras.2014.11.013

Santhi, K. and R.S.D. Wahida Banu, 2015. Adaptive contrast enhancement using modified histogram equalization. Optik Int. J. Light Electron Optics Elsevier, 126: 1809-1814.

DOI: $10.1016 /$ j.ijleo.2015.05.023

Singh, K., R. Kapoorand and S. Sinha, 2015. Enhancement of low exposure images via recursive histogram equalization algorithms. Int. J. Light and Electron Optics, Elsevier, 125: 1385-1389. DOI: $10.1016 /$ j.ijleo.2015.06.060 
Suganya, P., S. Gayathri and N. Mohanapriya, 2013. Survey on Image enhancement techniques. Int. J. Comp. Applica. Technol. Res., 2: 623-627.

DOI: 10.7753/IJCATR0205.1019
Xu, H., Q. Chen, C. Zuo, C. Yang and N. Liu, 2015. Range limited double-thresholds multi-histogram equalization for image contrast enhancement. Optical Rev. Springer, 22: 246-255.

DOI: $10.1007 / \mathrm{s} 10043-015-0073-\mathrm{x}$ 